\title{
Relic Abundance Analysis by Planckian Interacting Massive Particles and Weakly Interacting Massive Particles
}

\author{
Rakesh Sharma \\ National Defence Academy Khadakwasla Pune 411023 India
}

\begin{abstract}
There is one common point on which each astrophysicist believe strongly that most of the contained of our universe is dark and non baryonic. In cultivating recent results it is believe that promising candidates by whom the dark matter is made up are Weakly Interacting Massive Particles (supersymmetric candidate Neutralino) and Axions. A new model is proposed in which Planckian Interacting Massive Particles (PIDMs) are proposed to be the promising dark matter candidates [1]. In this paper we are analyzing the PIDMs with WIMPs to get relic abundance by both of models.
\end{abstract}

Keyword: Dark Matter, Gravitational Interaction

\section{Introduction}

Almost all astrophysicist now start to believe that most of the contain of our universe is non relativistic moving dark matter. Though direct detection techniques are still struggling to detect any signal of dark matter but enough observational evidences of its presence are available from long time. The gravitational lensing, rotational curves of spiral galaxies are major evidences of existence of dark matter [2]. In the big bang neucleosynthesis it was suggested that the baryon density is $\Omega b \lesssim 0.1[3]$ this amount is very less in the account of structure of universe. Neutrino of low mass like $30 \mathrm{eV}$ can fit well in providing right density of dark matter but is failed to explain structure formation in $\mathrm{N}$ body simulation [4]. Another problem is that they are moving with relativistic velocity hence will not match with non relativistic nature of dark matter.

\section{Wimps and Other Candidates}

The particle called Axion which arises from Peccei-Quinn solution of strong CP problem, Weakly Interacting Massive Particles arise from supersymmetric extension of standard model are found most promising dark matter till the new challenge of PIDMs arrive[1]. In this letter we are investigating characteristic nature WIMPs over PIDM. This will help us to understand nature of dark matter.

\section{Planks Parameters}

Let us check this out what exactly the Planck hypothesis. The Planck scale expresses that region in which the Standard Model(SM) and Quantum Field Theory (QFT) cannot be reconcilable. The parameters of Planck scale are:

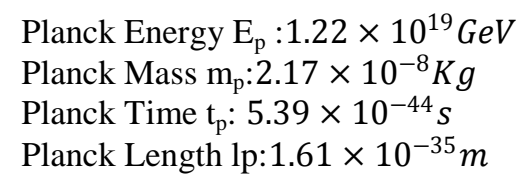

Other unit of charge and temperature are also available in literature we are not figuring them here.

\section{Mass of Wimps And Pidm}

The mass of WIMPs are in the range of few $\mathrm{GeV} / \mathrm{c}^{2}$ to thousand $\mathrm{Gev} / \mathrm{c}^{2}$ on the other hand PIDM candidate mass range from $10^{-3} \mathrm{M}_{\mathrm{p}}$ to $1 \mathrm{M}_{\mathrm{p}}$ where $1 \mathrm{M}_{\mathrm{p}}=1.2 \times 10^{19} \mathrm{GeV}$.In the Planckian scale the force of gravitation works differently as it is a manipulation of mathematics by removing the dimensions from fundamental forces like in this case it is considered by tacking $G=c=1$. It works in making the simple approach to mathematical complex equations but these manipulations have doubt many times [5]. We challenge the proposal of PIDM on the basis of rotational velocity and the relic abundance.

\section{Rotation Curve}

As it is believe that dark matter form a halo around the galaxies and the galaxy like ours, have density distribution of dark matter as:

$$
\rho_{(r)}=\rho_{0} \frac{r_{0}^{2}+a^{2}}{r^{2}+a^{2}}
$$

$\mathrm{r}=$ radius of halo, $\mathrm{r}_{0}=8.5 \mathrm{kpc}$ is distance of our galaxy from the center and a is core radius of halo. The halo gives rise to rotation curve velocity of galaxy:

DOI: $10.9790 / 4861-0805020104 \quad$ www.iosrjournals.org $\quad 1 \mid$ Page




$$
v_{h}^{2}(r)=4 \pi G \rho_{0}\left(r_{0}^{2}+a^{2}\right)\left[1-(a / r) \tan ^{-1}(r / a)\right]
$$

$\mathrm{G}$ is Newton's constant. At very large distances $r \gg 10 \mathrm{kpc}$ the velocity will be $220 \mathrm{~km} / \mathrm{s}$. At local position due to presence of disk

$$
v_{c}^{2}\left(r_{0}\right)=v_{d}^{2}\left(r_{0}\right)+v_{h}^{2}\left(r_{0}\right)
$$

where velocity due to disk $v_{d}\left(r_{0}\right)=118-115 \mathrm{~km} / \mathrm{s}$ andhalo contribute $v_{h}\left(r_{0}\right)=150-185 \mathrm{~km} / \mathrm{s}$ so local halo density is $0.3-0.5 \mathrm{GeV} / \mathrm{cm}^{3}$. The particles which makes dark matter halo by Virial theorem will moves with $220 \mathrm{~km} / \mathrm{s}$ velocity. As it is proposed in the PIDM model [1] there is no room for the rotation velocity because by using above equation one have to take this into account that $\mathrm{G}=1$ and by putting the other standard values we will not get the same results as we consider by taking WIMP into account. Thus for WIMPs dark matter will have density. The distribution of WIMPs dark matter is isotropic and distributed by Maxwell Boltzmann distribution. Hence disk and bulge of galaxy immersed in dark matter halo with local density $0.4 \mathrm{GeV} / \mathrm{cm}^{3}[6]$.

\section{Relic Abundance}

As per the Maxwell Boltzmann distribution of WIMP their current cosmological abundance is presented by:

$$
\Omega_{\chi} h^{2}=\frac{m_{\chi} n_{\chi}}{\rho_{c}} \simeq\left(\frac{3 \times 10^{-27} \mathrm{~cm}^{3} \sec ^{-1}}{\sigma_{A} v}\right)
$$

Here $\mathrm{h}$ is Hubble constant its unit is $100 \mathrm{kmsec}^{-1} \mathrm{Mpc}^{-1}$.It is shown that as the annihilation cross section increases WIMP stay in the equilibrium longer and the relic abundance decreases [7]. This will also suggest that the $\Omega_{\chi} \sim 1$ from above value comes from age of the universe and second and more important fact is that the interaction between the particles will be electroweak to have this density. It is the basis of ale the experimental efforts around the world in search of WIMP in the dark matter halo.

On the other hand the relic abundance of PIDM is given by :

$$
\Omega_{X} h^{2}=9.2 \times 10^{24} \gamma^{4 /(1+\omega)} \frac{m_{X}}{M_{p}} X_{f}
$$

Here $\gamma$ is the reheating parameter which must have value of the order of $10^{-3}$ for production of PIDM $[1,8]$. In the work of Garny et al.[1] shows that how cross sectional area did not affect the abundance it is the mass of particle which is responsible for relic abundance. Well the matter is debatable as in their work they have not express how do they co relate this formula with today age of universe or how it will be able to calculate $\Omega_{\chi}$ $\sim 1$ by this.

\section{Conclusion}

We had investigated the thermal relic density of WIMP and PIDM candidates. For the WIMP the figure 1 indicates that as the annihilation cross section increases the relic abundance decreases. Hence thermal relic density is insensitive to the mass of WIMP[9]. We had also investigated the effect of final abundance on relic density by keeping the value of $\gamma=1$ and considering variation in the value of $\mathrm{H}_{\mathrm{i}}$ by changing the mass. Figure 2 shows that as the final abundance $X_{\mathrm{f}}$ increases the relic abundance will increase [1]. This shows that it will not provide the relic density at present stage. Recently $\mathrm{T}$. Matsakos proposed idea of gravitational refraction to rule out the mysterious dark matter but he failed to explain mass to light ratio and his area of interest was disk shape of galaxies only hence have no answer for phenomenon like gravitational lensing [10].

[1]. M.Garny et al., PRL 116101302 (2016).

\section{Reference}

[2]. K.G. Begeman, A.H. Broeils, and R.H. Sanders, Mon. Not. R. Astr. Soc. 249, 523 (1991).

[3]. K.A. Olive, G. Steigman, D.N. Schramm, T.P. Walker, and H. Kang, Astrophys. J. 376, 51 (1991).

[4]. S.D.M.White, C.S. Frenk, andM. Davis, Astrophys. J. 274, L1-5 (1983)

[5]. P.S.Wesser, Sp.Sc. Rev.27(2),117(1980).

[6]. M.Kamionkovski,A.Kinkhabwala (hep-ph/9710337)

[7]. M,Kamionkovski,(1997) hep-ph/9710467.

[8]. G. Felder, L. Kofman, and A. Linde, Phys. Rev. D 59, 123523 (1999).

[9]. J.L. Feng,arXiv:1003.0904 [astro-ph.CO](2010)

[10]. T.Matsakos, A.Diaferio "Dynamics of galaxies and clusters in refracted gravity" arXiv:1603.04943v1[astro-ph.GA] 16 Mar 2016. 
Figures Of Results

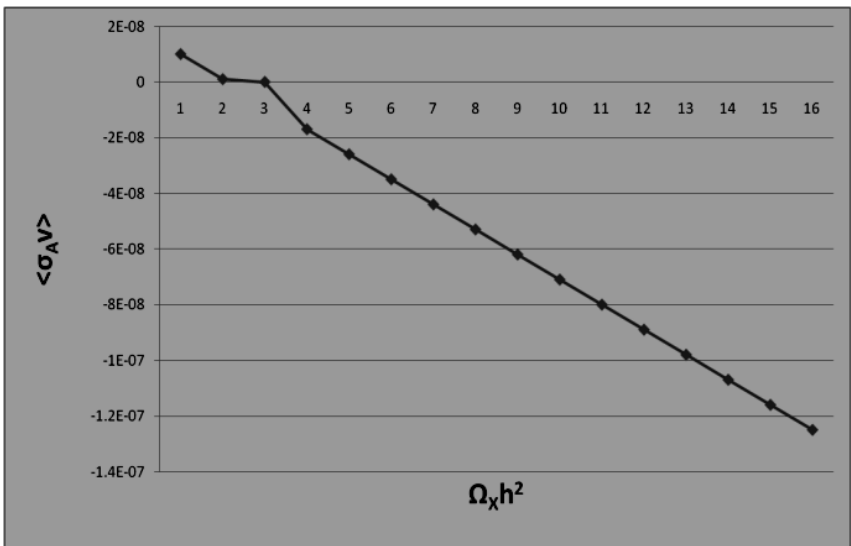

Figure 1: The graph shows that relic abundance depends on area of cross section and is independent of mass of WIMP. The calculations are based on data presented in [7].

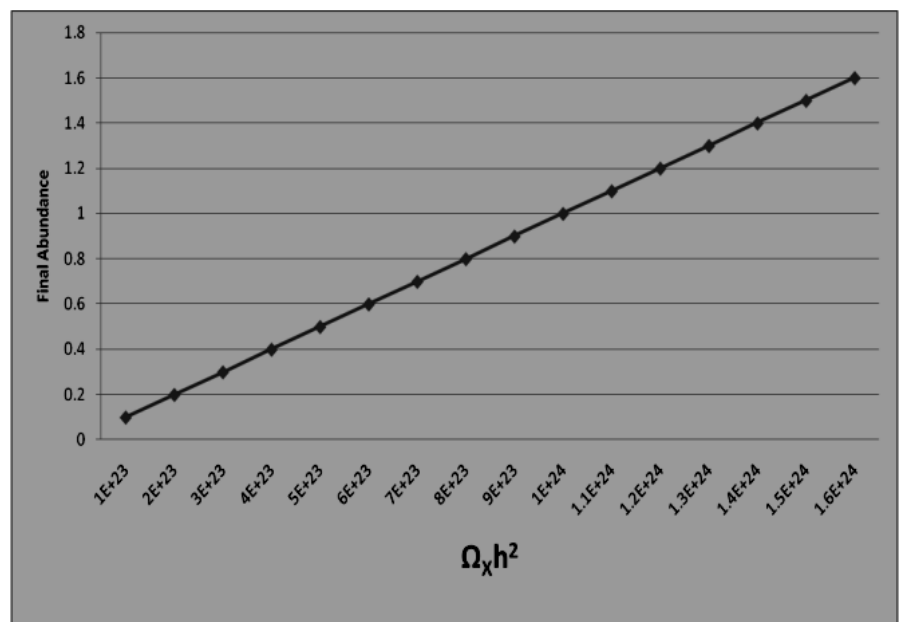

Figure 2: As it is established in the phenomenon of PIDM that relic abundance is affected by the mass of dark matter candidates proposed in PIDM hence by taking value of $\gamma=1$ and considering variation in the value of $H_{i}$ by changing the mass we get that relic abundance will increase as mass increases. The calculation is based on the parameters and data given in [1].

\section{APPENDIX}

The mass and cross sectional area of interaction relation.

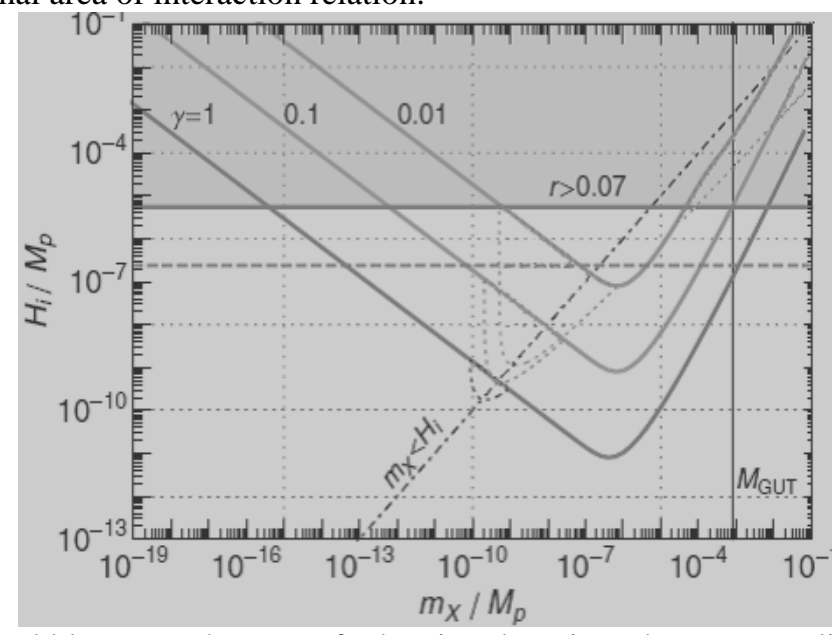

FIG. 3. The value of the Hubble rate at the start of reheating that gives the correct relic abundance, as a function of the mass of the PIDM. The curve for $\gamma=1, \gamma=0.1$, and $\gamma=0.01$. The dotted lines show the modification when taking also gravitational production into account. The dashed-dotted line marks $\mathrm{m}_{\mathrm{X}}=\mathrm{H}_{\mathrm{i}}$. All values are given in units of $\mathrm{M}_{\mathrm{p}}[1]$ 


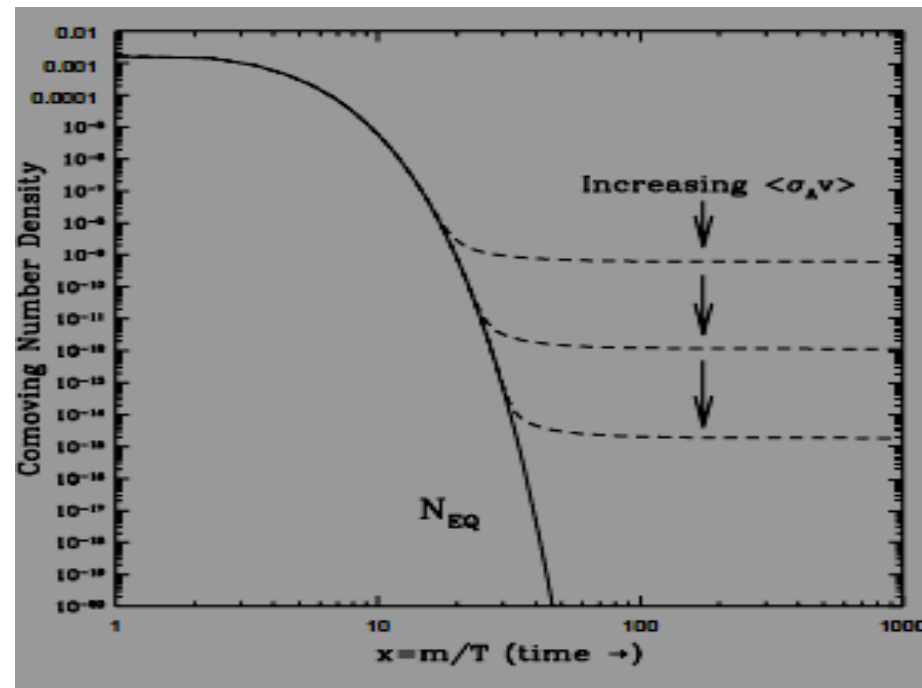

Figure 4: Comoving number density of a WIMP in the early Universe. The dashed curves are the actual abundance, and the solid curve is the equilibrium abundance[7]. 